\title{
Treatment of Grade II Mandibular Furcation Defects by Concentrated Growth Factors and Hydroxyapatite Bone Substitute (Sybograf ${ }^{\circledR}$ ): A Case Report
}

\author{
${ }^{1}$ Senthil Rajan, ${ }^{2}$ Kavita Chandrasekaran, ${ }^{3}$ Krishna Kripal, ${ }^{2}$ Thaslim Fathima
}

\begin{abstract}
Aim: This case report attempts an evaluation of the clinical and radiographic outcome of concentrated growth factors (CGF) along with nanohydroxyapatite bone substitute in considering grade II mandibular furcation defects.
\end{abstract}

Background and objectives: Periodontal disease, if not interfered, will headway further with attachment loss, adequate enough to have a consequence on the bifurcation or trifurcation of multirooted teeth. There is a paucity of studies comparing the effects of CGF and nanohydroxyapatite crystals bone substitute in furcation defects.

Case discussion: A male patient, 66 years old, had visited the outpatient department of periodontology, with a chief complaint of sensitivity in the lower left back tooth region for the past few months. The probing depth as measured by UNC-15 probe was $5 \mathrm{~mm}$ mesiobuccal, $10 \mathrm{~mm}$ midbuccal, $5 \mathrm{~mm}$ distobuccal and $5 \mathrm{~mm}$ midlingual. The furcation defect was filled using an appropriate combination of CGF and bone graft material (Sybograf). The flap was sutured with 3-0 silk suture to obtain primary closure.

Conclusion: The results have shown significant improvement in the gingival index and probing pocket depth (horizontal and vertical). There was a significant amount of bone fill also that was evident radiographically.

Clinical significance: The current case report boons a new approach for the treatment of grade II mandibular furcation defects through a collective approach of CGF and nanohydroxylapatite bone substitute.

Keywords: Concentrated growth factor, Furcation involvement, Hydroxyapatite crystals, Periodontal disease.

How to cite this article: Rajan S, Chandrasekaran K, Kripal K, Fathima T. Treatment of Grade II Mandibular Furcation Defects by Concentrated Growth Factors and Hydroxyapatite Bone Substitute (Sybograf ${ }^{\circledR}$ ): A Case Report. J Health Sci Res 2018;9(2):61-65.

\section{Source of support: Nil}

Conflict of interest: None

\footnotetext{
${ }^{1}$ Reader, ${ }^{2}$ Postgraduate Student, ${ }^{3}$ Professor

${ }^{1-3}$ Department of Periodontology, RajaRajeswari Dental College and Hospital, Bengaluru, Karnataka, India

Corresponding Author: Kavita Chandrasekaran, Postgraduate Student, Department of Periodontology, RajaRajeswari Dental College and Hospital, Bengaluru, Karnataka, India, e-mail: dr.ckavita@gmail.com
}

\section{INTRODUCTION}

Periodontitis is a multifactorial disease which begins as an inflammation of periodontium. As the disease progresses, it causes the destruction of the supporting tissues which is clinically reflected as a loss of attachment. Multiple modalities of treatment have been performed clinically resolve furcation defects some of which include autografts, demineralized freeze-dried bone allografts, bovine-derived xenografts, barrier membranes and various combinations of these. The fear of chance of disease transmission and the lack of adequate donor materials have limited the use of allografts and autogenous grafts on a routine basis. This led to the development of alloplasts or synthetic bone substitutes for periodontal applications. ${ }^{1}$

One such synthetic material is the nanohydroxyapatite crystal bone substitute (Sybograf $®$ ) has been presented for the augmentation procedures in periodontal bony defects due to its reported advantages of osteoconductivity, bioresorbability and close contact with the resorbed bony surface. Another distinct feature of such nanostructured materials is an exceptionally high number of molecules on the surface of the material. It adheres to the bone and therefore encourages bone healing by stimulating osteoblastic activity. $^{2}$

Recent reports in the literature have demonstrated that nanohydroxyapatite crystals (Sybograf ${ }^{\circledR}$ ) may support regeneration in class II furcation defects by its overstimulating mode of action. It has been a great improvement in clinical indices for mandibular molar class II furcation treated with Sybrograf ${ }^{\circledR}$ as a bone replacement graft than in those treated with open flap debridement surgery. ${ }^{3}$

Although these synthetic regenerative materials are still used, the introduction of biomimetic agents like enamel matrix derivatives, bone morphogenic proteins, and platelet-derived growth factor (PDGF) have been used more recently for better outcomes in the management of furcation defects. ${ }^{3}$

Concentrated growth factor (CGF) represents a new group of platelet concentrates, which have plentiful growth factors. ${ }^{4}$ 
Preparation of CGF is carried out by centrifuging the venous blood. It forms a layer platelet concentrate along with the brin matrix which is similar to platelet-rich-fibrin (PRF). However, the speed of centrifugation differs which in turn allows the segregation of much denser, larger and richer growth factors (GFs) in the fibrin matrix of CGF as compared to that of PRF. This mass has a property of high cohesion due to the agglutination of factor XIII, brinogen, and thrombin. This, in turn, prevents plasmin degradation, thereby resulting in high stability and fibrin tensile strength. Recently, various researchers have suggested the use of CGF in the reconstruction of the bony defects. They observed from their studies that bone formation significantly increased with the use of CGF. ${ }^{5}$

There is a paucity of research comparing the effects of CGF and nanohydroxyapatite crystals bone substitute in furcation defects. Therefore, this case report attempts an appraisal of the clinical and radiographic outcome of CGF along with nanohydroxyapatite bone substitute in treating mandibular grade II furcation defects.

\section{MATERIALS AND METHODS}

The patient was selected from outpatient department of periodontology, RajaRajeswari Dental College and Hospital, Bengaluru, Karnataka, India. The procedure and purpose of using CGF were clearly described to the patient and written informed consent was provided by the patient before the surgical treatment.

\section{Preparation of Concentrated Growth Factor}

Intravenous blood was collected in two $10 \mathrm{~mL}$ sterile tubes devoid of any anticoagulant solution and was placed in the centrifuge tubes. It was then accelerated for 30 seconds, centrifuged at $2700 \mathrm{rpm}$ for 2 minutes, 2400 rpm for 4 minutes, $2700 \mathrm{rpm}$ for 4 minutes, and $3000 \mathrm{rpm}$

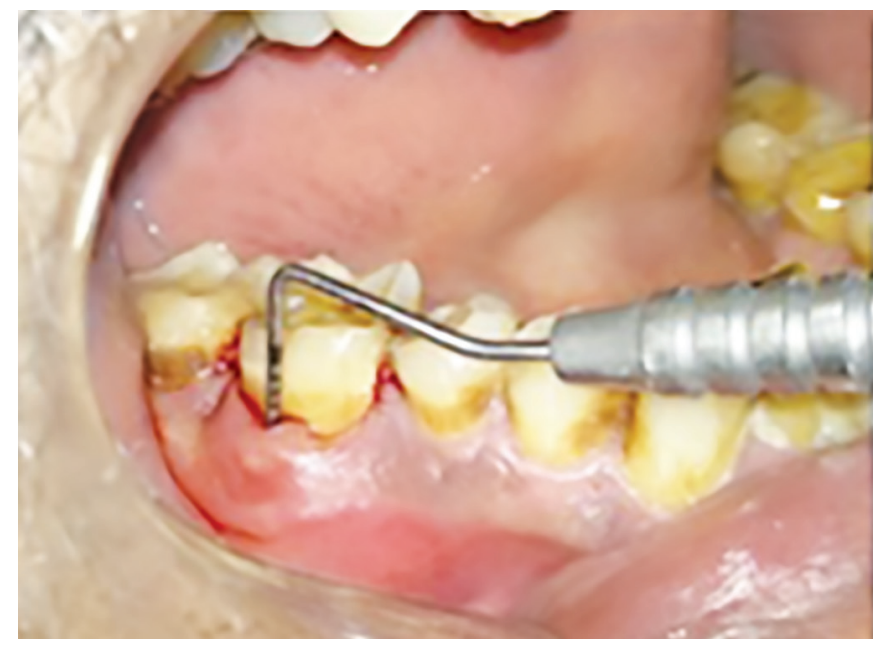

Fig. 1: Preoperative probing with UNC-15 for 3 minutes, and decelerated for $36 \mathrm{~s}$ to stop. At the end of the centrifugation, four blood fractions were observed: (1) the upper layer-this consists of mainly serum, (2) the second layer-this resembles a buffy coat, (3) the third layer-this is rich in growth factors (GF) and unipotent stem cells (CGF), and (4) the lower layer-consists of red blood cells (RBC). The obtained CGF clot was split from the RBC layer by using microsurgical scissors.

\section{CASE REPORT}

A 66-year-old male had visited the Department of Periodontology, at RajaRajeswari Dental College and Hospital, Bengaluru, Karnataka, India with a chief complaint of sensitivity in the lower left back tooth region for the past few months.

Upon clinical examination, the oral hygiene status was fair. There were gingival inflammation and abscess in relation to the buccal aspect of mandibular right first molar (46). The probing depth as measured by UNC-15 probe was $5 \mathrm{~mm}$ mesiobuccal, $10 \mathrm{~mm}$ midbuccal, $5 \mathrm{~mm}$ distobuccal and $5 \mathrm{~mm}$ midlingual. The horizontal probing depth as measured by Naber's probe was $9 \mathrm{~mm}$ (Figs 1 and 2). These clinical findings were supported by RVG taken at baseline which showed a significant amount of bone loss in relation to the same tooth. Clinical parameters were recorded at baseline, 3 months and 6 months-gingival index, horizontal and vertical probing pocket depths and CAL.

Phase I therapy was performed which comprised complete supragingival, subgingival scaling and root planing. Detailed plaque control instructions were given and the patient was recalled after one week for a recall check-up. At the recall visit the probing pocket depths reduced to $3 \mathrm{~mm}$ on mesiobuccal and distobuccal sites whereas it remained the same at the midbuccal and midlingual sites. Thus, it was decided to perform a surgical intervention to treat the mandibular furcation defect.

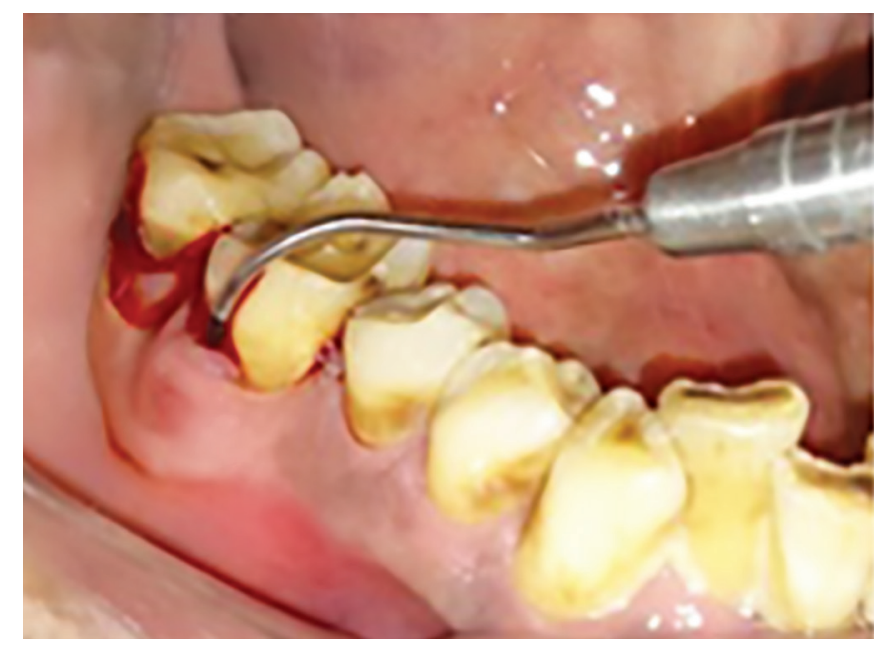

Fig. 2: Preoperative probing with Naber's probe 


\section{Treatment Procedure}

Intraoral antisepsis was achieved using $0.12 \%$ chlorhexidine digluconate rinse. Following administration of LA, a \#15 blade was used to place incision and a full-thickness flap was reflected. Following this, the granulation tissue was removed and complete debridement was done using

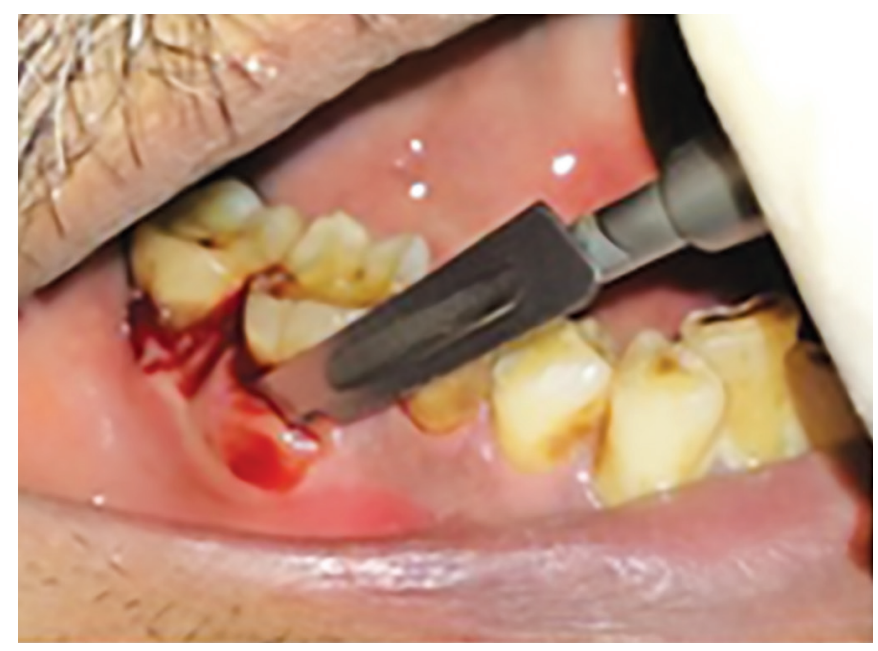

Fig. 3: Incision with \#15 blade

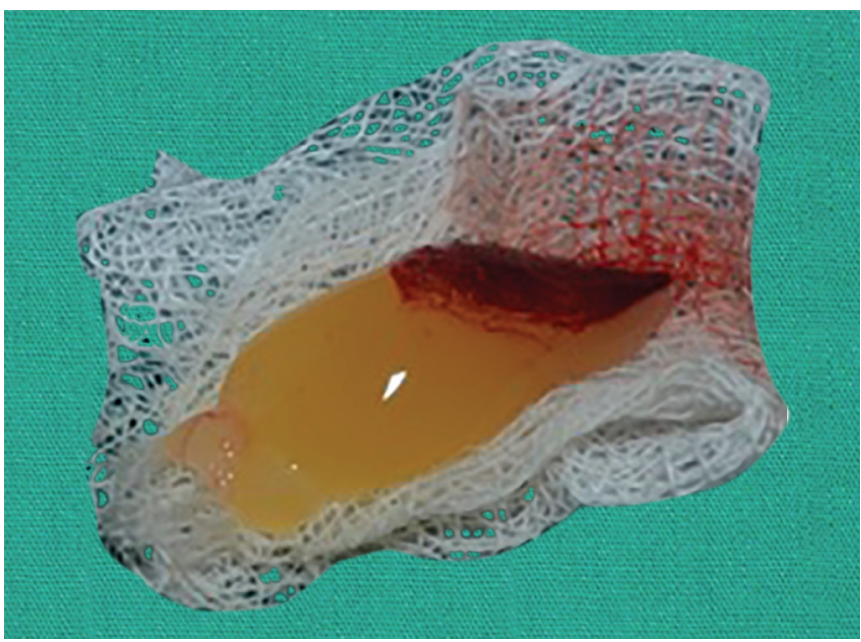

Fig. 5: Concentrated growth factor

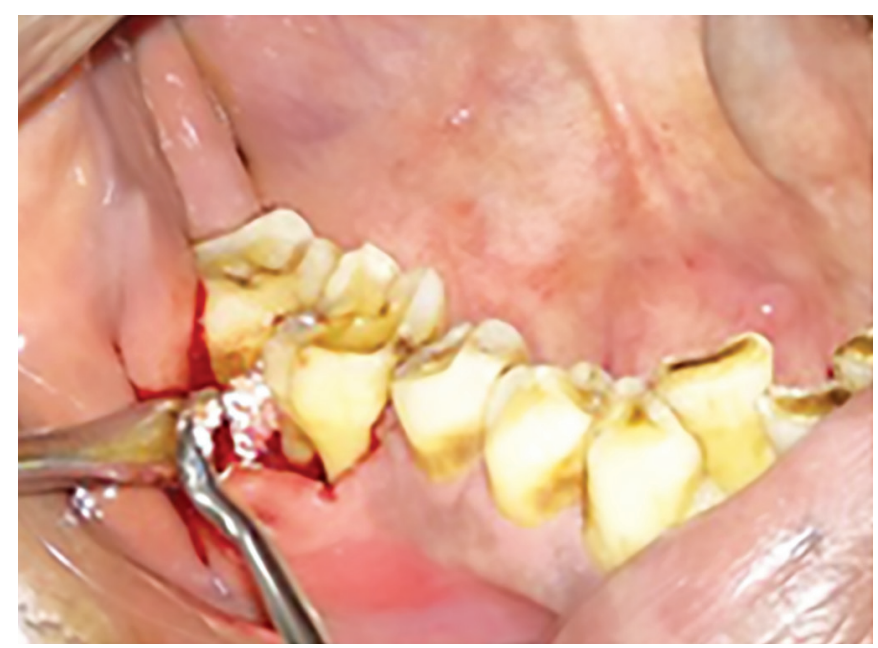

Fig. 7: Placement of CGF with sybograf ${ }^{\circledR}$
Gracey curettes. After presuturing, the furcation defect was filled using the appropriate combination of CGF and bone graft material (Sybograf). The flap was sutured with 3-0 silk suture to obtain primary closure and noneugenol periodontal dressing (Coe pack) was placed (Figs 3 to 10).

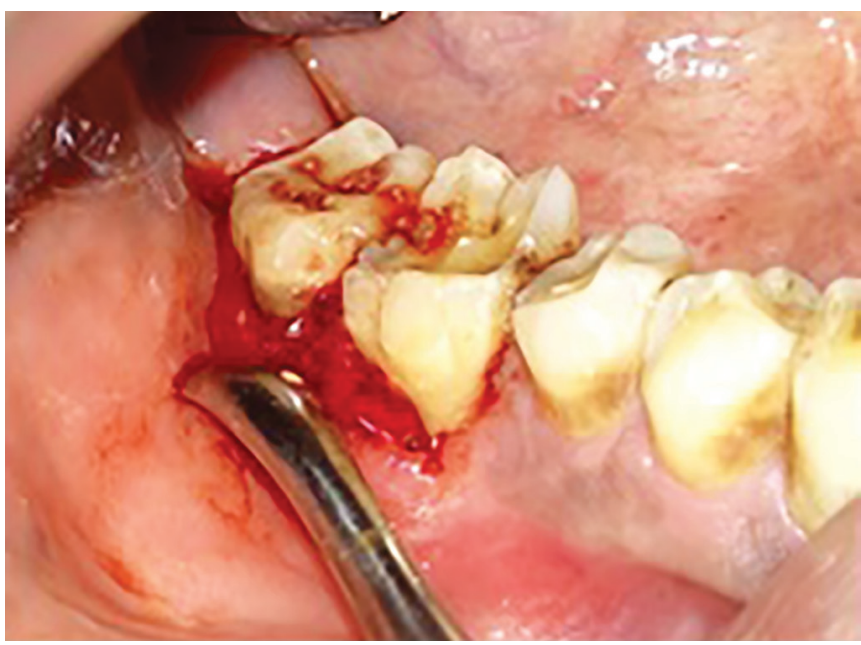

Fig. 4: Reflection and debridement

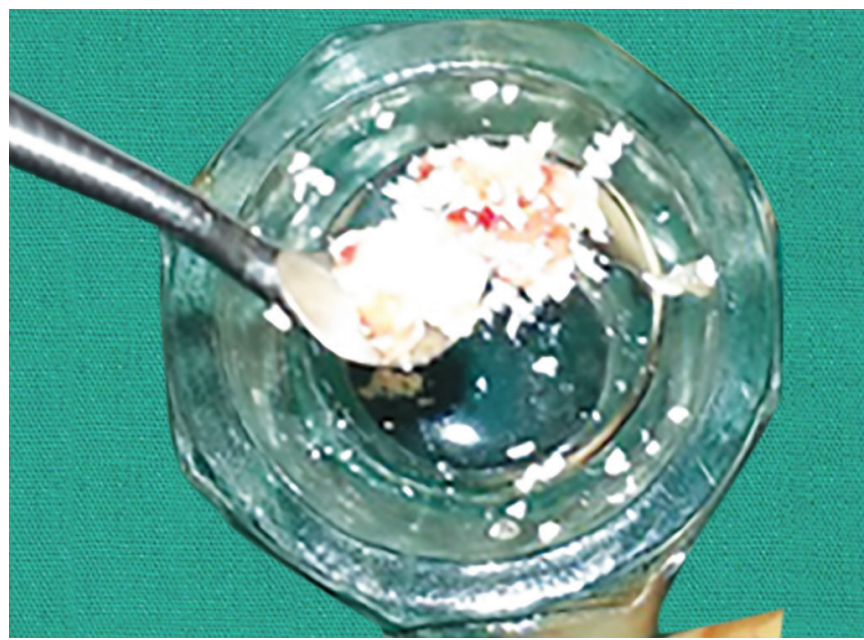

Fig. 6: Concentrated growth factor combined with Sybograf $®$

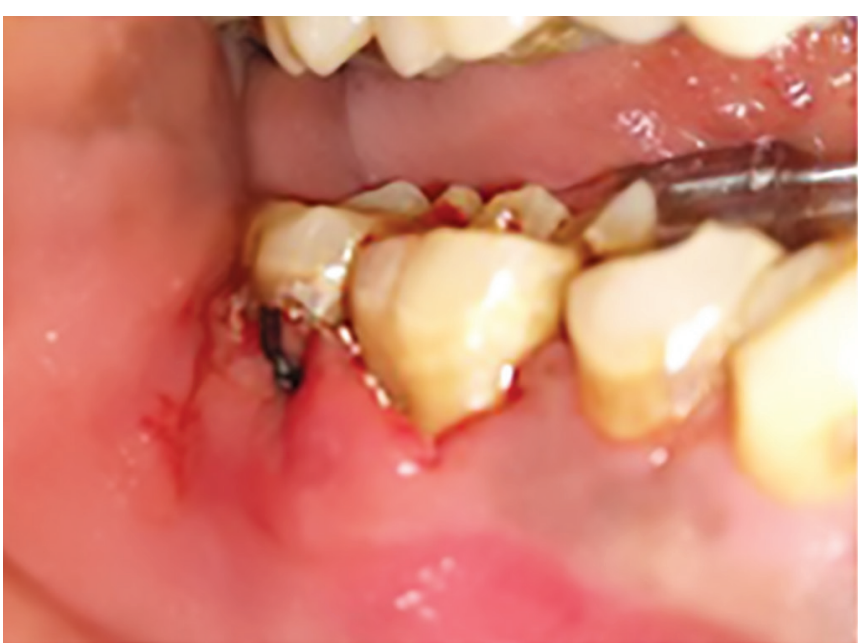

Fig. 8: Sutures placed 


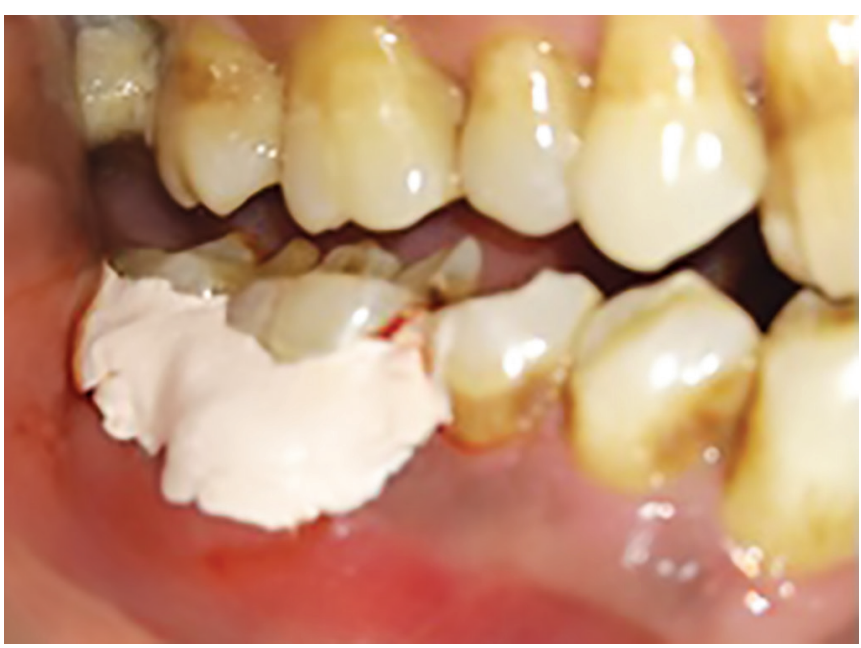

Fig. 9: Coe-pack placed

\section{Postoperative Care}

Suitable antibiotic and analgesics were prescribed. The patient was advised to rinse with chlorhexidine digluconate $(0.12 \%)$ twice daily for 2 weeks following surgery. Periodontal dressing and sutures were removed 2 weeks postoperatively. The patient was recalled after 3 and 6 months for a review. An RVG was taken at 6 months to assess bone regeneration (Figs 11 and 12).

\section{RESULTS}

There was a noticeable enhancement in all the clinical parameters. The radiographs obtained at baseline and 6 months showed marked bone fill (Table 1).

Table 1: Clinical parameters and level of bone measured from the radiograph

\begin{tabular}{|c|c|c|c|c|c|}
\hline & $\begin{array}{l}\text { Gingival } \\
\text { index }\end{array}$ & $\begin{array}{l}\text { Horizontal } \\
\text { probing } \\
\text { pocket } \\
\text { depth }\end{array}$ & $\begin{array}{l}\text { Vertical } \\
\text { probing } \\
\text { pooket } \\
\text { depth }\end{array}$ & $C A L$ & $\begin{array}{l}\text { Bone } \\
\text { level in } \\
\text { RVG }\end{array}$ \\
\hline At baseline & 2.3 & $9 \mathrm{~mm}$ & $10 \mathrm{~mm}$ & $10 \mathrm{~mm}$ & $8 \mathrm{~mm}$ \\
\hline 3 months & 1.5 & $6 \mathrm{~mm}$ & $8 \mathrm{~mm}$ & $8 \mathrm{~mm}$ & $8 \mathrm{~mm}$ \\
\hline 6 months & 1.2 & $6 \mathrm{~mm}$ & $5 \mathrm{~mm}$ & $5 \mathrm{~mm}$ & $5 \mathrm{~mm}$ \\
\hline
\end{tabular}

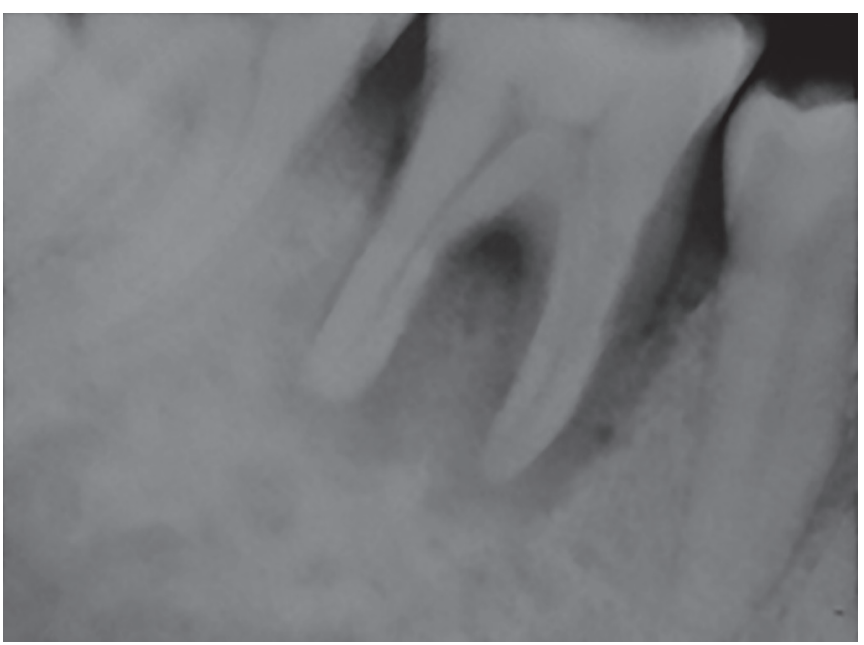

Fig. 11: Preoperative RVG

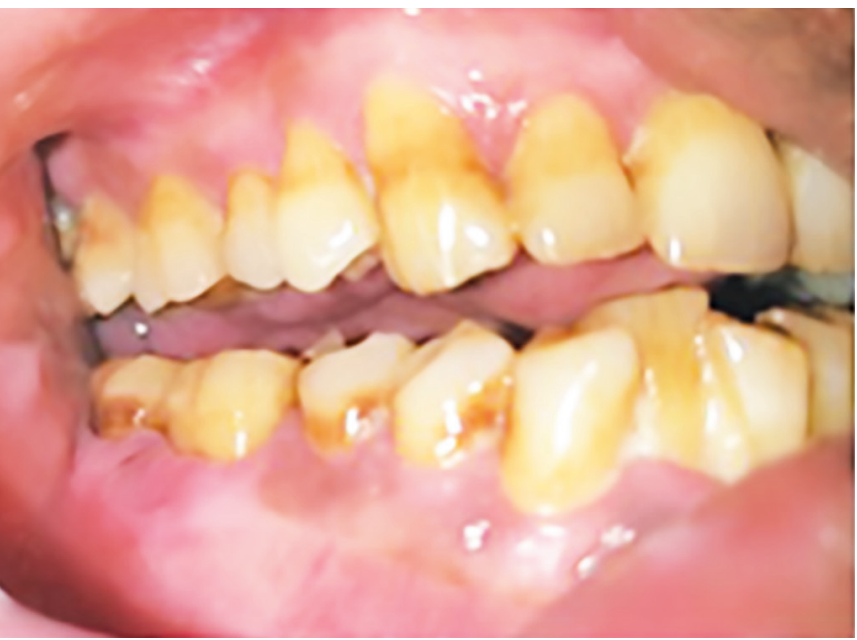

Fig. 10: Postoperative at 6 months

The vertical probing depth had reduced from $10 \mathrm{~mm}$ at baseline to $5 \mathrm{~mm}$ at 6 months and the horizontal probing reduced from $9 \mathrm{~mm}$ at baseline to $6 \mathrm{~mm}$ at 6 months. Also, the level of bone (from cementoenamel junction to the deepest point of the defect as seen in RVG) had improved from $8 \mathrm{~mm}$ at baseline to $5 \mathrm{~mm}$ at 6 months.

\section{DISCUSSION}

The principal objective of periodontal regeneration is to re-establish the hard and softtissue components of the periodontium, including the formation of a new attachment apparatus, which results in the closure of the furcation. There is an ample number of regenerative approaches which have been applied in patients with class II furcation involvements. Some of these include root surface biomodification, coronally positioned flaps, various bone grafts, and other combination procedures.

In a study by Bajaj et al. in 2013, the results of the treatment of class II furcation defects in the mandible with either autologous PRF with open ap debridement or autologous PRP with open flap debridement (OFD),

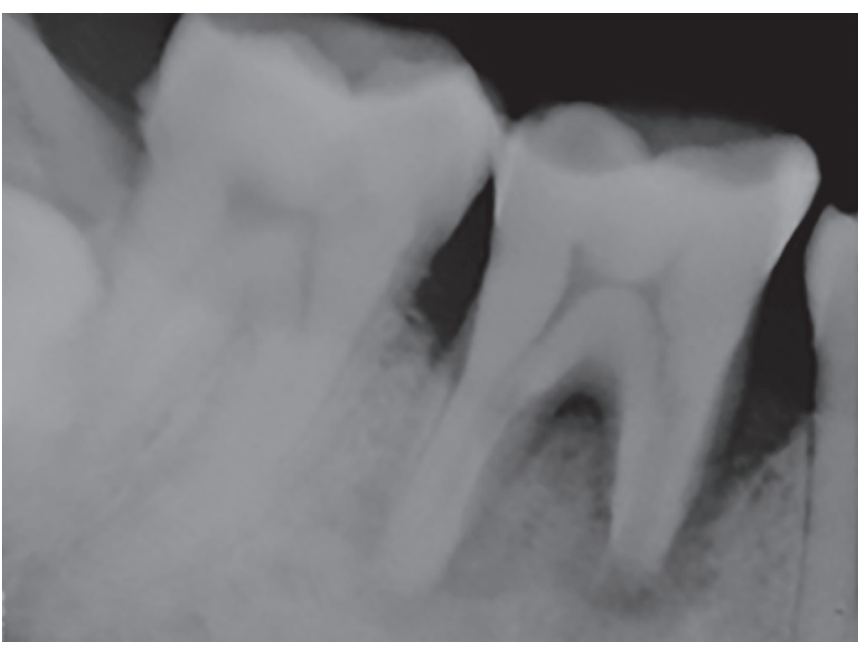

Fig. 12: Postoperative RVG 
or only OFD was evaluated in 72 mandibular class II furcation defects in patients with chronic periodontitis. Clinical parameters such as clinical attachment level, probing pocket depth, horizontal CAL and gingival marginal level were estimated at baseline and at 9 months. All the parameters improved significantlyin both the test sites (PRF with OFD and PRP with OFD) compared to those with OFD alone and the study concluded that the application of both autologous PRP/PRF was equally effective in the management of furcation defects. ${ }^{4}$

A recent study was carried out by Qiao et al. in 2016 to study the efficacy of CGF in the management of vertical bony defects. In this study, 31 defects were included and managed with either CGF + bovine porous bone mineral (BPBM) or BPBM alone. Clinical attachment level, probing depth, and bone fill was assessed parameters. Both groups presented noteworthy enhancement in clinical parameters $(p<0.001)$. CGFs + BPBM was more efficient than BPBM alone at subsiding pocket depth $(4.2 \pm 1.3 \mathrm{~mm}$ vs. $3.0 \pm 1.6 \mathrm{~mm})$ and CAL gain $(3.7 \pm 1.3 \mathrm{~mm}$ vs. $2.4 \pm 1.1 \mathrm{~mm} ; p \leq 0.05)$ at 1 year. A favorable escalation of hard tissue fill was seen in CGFs + BPBM group when equated with BPBM group $(p>0.05)$. The study determined that the use of CGF significantly improved the effectiveness of BPBM in the cure of periodontal bony defects. ${ }^{5}$

Clinical research was conducted by Dogan et al. in 2015 to gauge the practical efficacy of concentrated growth factor (CGF) for management of multiple adjacent gingival recessions (GRs), in amalgamation with coronally advanced flap (CAF) and CAF alone. For this study, a total of 119 Miller class I and II GRs in the maxilla of 20 patients were evaluated. At the end of 6 months, they found an escalation in dimensions of keratinized gingiva in terms of its width (KGW) and gingival thickness (GT) which were statistically significant in the CAF + CGF group when compared to the CAF only group. The study concluded that combining CGF with CAF might result in better coverage of root due to the significant increase in KGW and GT. ${ }^{6}$

Pirpir et al. conducted a study in 2017 to evaluate the efficiency of the concentrated growth factor on osseointegration and implant stability. For this study, 12 patients with the maxillary anterior edentulous area were recruited. Implant cavities were concealed with CGF membranes before implant placement. An Osstell device was incorporated into the intraoperative procedure to measure resonance frequency. This measurement was taken at 1 st and 4 th weeks. Positive effects on implant stabilization were observed due to the concentrated growth factor. The implant stability quotient (ISQ) measurements at the 1st and 4th weeks were significantly greater in the study group and use of CGF seems to hasten the osseointegration. ${ }^{7}$

In the present case, combined treatment approach using nanohydroxyapatite bone substitute and CGF was used for management of mandibular grade II furcation defect. The CGF used provides an advantage over PRF due to greater, denser and increased number Growth Factors (GFs) in a brin matrix which could have contributed to enhancement in the clinical outcomes and radiographic bone fill.

\section{CONCLUSION}

Within the limitations, the current clinical case highlights a new modality for management of mandibular grade II furcation defects through a combined approach of CGF and nano hydroxyl apatite bone graft. The results have shown substantial enhancement in the clinical parameters of the gingival index and probing pocket depth (horizontal and vertical). There was a significant amount of bone fill also that was evident radiographically. Thus, this modality could prove to be a boon for treating mandibular furcation defects.

\section{REFERENCES}

1. Asmita, Gupta V, Bains VK, Singh GP, Jhingran R. Clinical and cone beam computed tomography comparison of NovaBone Dental Putty and PerioGlas in the treatment of mandibular Class II furcations. Indian J Dent Res 2014;25:166-173.

2. Singh VP, Nayak D, Uppoor AS, Shah D. Clinical and radiographic evaluation of Nano-crytalline hydroxyapatite bone graft $\left(\right.$ Sybograf $\left.{ }^{\circledR}\right)$ in combination with bioresorbable collagen meme $=$ brane $\left(\right.$ Periocol $\left.^{\circledR}\right)$ in periodontal intrabony defects. Dent Res J (Isfahan) 2012;9(1):60-67.

3. Verma PK, Srivastava R, Gupta KK, Chaturvedi TP. Treatment strategy for guided tissue regeneration in various class II furcation defect: Case series. Dent Res J (Isfahan) 2013:10(5): 689-694.

4. Bajaj P, Pradeep AR, Agarwal E, Rao NS, Naik SB, Priyanka $\mathrm{N}$, et al. Comparative evaluation of autologous platelet-rich fibrin and platelet-rich plasma in the treatment of mandibular degree II furcation defects: a randomized controlled clinical trial. J Periodont Res 2013; 48:573-581.

5. Qiao J, Jinyu Duan, Zhang Y, Chu Y, Sun C. The effect of concentrated growth factors in the treatment of periodontal intrabony defects. Future Science OA. 2016;2:4.

6. Dogan SB, Dede FO, Balli U, Atalay EN, Durmuslar MC. Concentrated growth factor in the treatment of adjacent multiple gingival recessions: a split-mouth randomized clinical trial. J Clin Periodontol 2015;42:868-875.

7. Pirpir C, Yilmaz O, Candirli C, Balaban E. Evaluation of effectiveness of concentrated growth factor on osseointegration. Int J implant Dent. 2017;3:7. 